www.jmscr.igmpublication.org

Impact Factor (SJIF): 6.379

Index Copernicus Value: 71.58

ISSN (e)-2347-176x ISSN (p) 2455-0450

crossrefDOI: https://dx.doi.org/10.18535/jmscr/v6i7.138

Journal Of Medical Science And Clinical Research

\title{
Original Paper \\ Study of Small Dense Low Density Lipoprotein Cholesterol, Paraoxonase1, and Lipid Profile in Postmenopausal Women
}

\author{
Authors \\ Dr Shyam Khanderao Kulkarni ${ }^{1}$, Dr Abhay John ${ }^{2^{*}}$, Dr Rahul Kunkulol ${ }^{3}$ \\ Dr Sayyed Asif Umar ${ }^{4}$ \\ ${ }^{1}$ Assistant Professor, Department of Biochemistry PMT, PIMS \\ ${ }^{2}$ Assistant Professor, Department of Pharmacology PMT, PIMS \\ ${ }^{3}$ Professor, Department of Pharmacology PMT, PIMS \\ Corresponding Author \\ Dr Abhay John \\ Assistant Professor, Department of Pharmacology PMT, PIMS
}

\begin{abstract}
The present study were, to estimate the small dense $L D L$ ( $s d L D L)$ cholesterol levels and the Paraoxonase A in postmenopausal women and to correlate the sdLDL-C levels, PON1 activity with menopause. Materials and methods; Present study was carried out in a medical college hospital. Total 40 postmenopausal women were enrolled as cases. These cases were compared with 40 healthy premenopausal women. The parameters studied were small dense LDL-C, paraoxonsel activity, and lipid parameters (Total cholesterol, triglyceride, $H D L-C, L D L-C$, and VLDL-C). Results and observation; In present study there is increased concentration of serum triglyceride $(167.52 \pm 68.79 \mathrm{vs} .118 .95 \pm 43.41 \mathrm{mg} / \mathrm{dl})$ which is proven independent risk factor for atherosclerosis. The decreased HDL-C concentration (30.92 $\pm 7.47 \mathrm{vs} .36 .67 \pm 7.94 \mathrm{mg} / \mathrm{dl})$ suggest altered lipoprotein pattern. The increased concentration of TG is associated with increased sdLDL with no change in LDL-C levels suggesting the menopause related qualitative changes in lipoprotein metabolism. The presence of atherogenicsd LDL with hypertriglyceridemia in postmenopausal women suggest the enhanced lipid transfer reaction, leading to increased synthesis of triglyceride rich and cholesterol poor sdLDL. The significantly increased concentration of sdLDL-C (62.87 $\pm 17.90 v s .34 .25 \pm$ $14.39 \mathrm{mg} / \mathrm{dl}$ ) in postmenopausal women than premenopausal suggest greater atherosclerotic tendency as sdLDL have greater oxidative susceptibility, tendency to accumulate in circulation and lower affinity for LDL receptors. Paraoxonase1, the HDL associated, antioxidant and protective enzyme is found to be low (116.16 \pm 76.59 vs. $206.55 \pm 69.05 U / L)$ in55postmenopausal women suggestive of decreased antioxidant defense after menopause. The decreased activity of PON1 could be due to lower levels of HDL-C. The decreasing PON1 activity was also found to relate with increased $s d L D L$.
\end{abstract}

\section{Introduction}

India, where most of the population is rural, experiencing a huge burden of cardiovascular diseases (CVD). In 2000 CVD accounted for $32 \%$ of all deaths and world health organization (WHO) estimates that $60 \%$ of the world's CVD patients will be Indian by $201{ }^{(1)}$. In India nearly 1.5 million people die of CVD each year. India is 
suffering from a heavy loss of productive age group as the total burden of CVD is increasing. This is expected that by the year 2030 the loss due to CVD rise to 17.9 million people per year which is 9.4 times greater than that of USA ${ }^{(2)}$.

The initial event which leads to the CVD is atherosclerosis. Atherosclerosis is a multistep, slowly evolving process. The process of atherosclerosis had started long before its consequences results. Studies have identified a number of important risk factors for atherosclerosis, including obvious one like male sex and increasing age. Some of the factors are modifiable and other non-modifiable.

\section{Non modifiable}

The non-modifiable factors are age, sex, family history and genetic factors. There is proven gender difference in the pattern of CVD, as the incidence of CVD is more in men than women, due to which for many years it was thought to be a men's disease. The CVD is the leading cause of mortality in women being responsible for 5 lacs deaths per year in USA, which is twice that of deaths due to cancer. It is also found that coronary artery disease is the single largest cause of deaths in postmenopausal women. ${ }^{(3)}$ There are specific age related gender differences. The onset of CHD is delayed by some $10-15$ years in women compared to men; thus ATP III defines age as a risk factor in women at age 55, compared to age 45 for men ${ }^{(4)}$. The reasons for the disparity in ages of onset of CHD between women and men are not fully understood. Nonetheless, patterns of risk factors often differ between men and women. For example, blood pressure, LDL cholesterol, and triglycerides, rise at an earlier age in men than in women. Moreover, HDL-cholesterol levels are on average some $10 \mathrm{mg} / \mathrm{dl}$ lower in adult men than in women. Since a $10-\mathrm{mg} / \mathrm{dL}$ difference in HDL cholesterol is projected to account for a 20-30 percent difference in CHD event rates over the short term, this difference over the adult lifespan could account for a significant portion of the gender disparity between men and women. The menopausal transition is proved to be associated with decrease in HDL-Clevels. Furthermore the menopause in itself a risk factor for atherosclerosis

There is increased prevalence of hypertension and dyslipidemia after menopause. ${ }^{(6)}$ The lipoprotein profile of postmenopausal women is characterized by increased levels of low density lipoprotein (LDL), Lp(a), triglyceride, total cholesterol, and decreased high density lipoprotein (HDL) cholesterol levels. Moreover, triglycerides appear to be an even more powerful and independent risk factor in women than in men. The lipoprotein pattern of postmenopausal women characteristically shows predominance of specific LDL subclass, small dense LDL (sdLDL). It is proved that as age advances the level of serum sdLDL particles also increases in women. ${ }^{(7)}$ A commonly cited reason for the gender difference is a protective effect of estrogen in women ${ }^{(8,9)}$. Oral estrogens increase HDL cholesterol and decrease LDL cholesterol. Oral estrogens do not mimic the physiologic role of endogenous estrogen, which is released into the systemic rather than the portal circulation $^{(10)}$. The anti-atherosclerotic effect of estrogen is probably due to its beneficial influence on lipid metabolism. The estrogen has an antioxidant activity. Thus, after menopause there may be increase in lipidper oxidation and formation of reactive oxygen species. This increased4oxidative stress may lead to endothelial injury and increased lipoprotein oxidation including HDL. This ultimately leads to accumulation of oxidized LDL in the sub endothelial space leading to atherosclerosis ${ }^{(11)}$. In premenopausal women, estrogen is involved in the inhibition of apoptosis. ${ }^{(12)}$ Thus, uncontrolled apoptosis may be the reason for increased CHD risk in postmenopausal women. Other effects which are related to estrogen are inhibition of endothelial hyperplasia, reduced arterial impedance, enhanced production of prostacyclins and increased insulin sensitivity. ${ }^{(12)}$ 


\section{Paraoxonase 1}

The purified serum $P O N 1$ have a molecular mass of $43-45 \mathrm{kDa}$ and contains as many as three carbohydrate chains accounting for $15.8 \%$ of the weight. PON 1 is synthesized and secreted by liver. PON1 activity is present in newborn and premature infants at about half that of adult. The PON 1 level and activity increases up to 7 years of age. ${ }^{(27)}$ Adult level reaches approximately 7year after the birth and remains constant throughout life.PON1 is associated with HDL particles and is believed to contribute to anti-atherogenic properties of HDLs. (28) PON 1 is anchored to HDL through apo A1 and it is extremely difficult to remove apo A1 from PON 1 during10 purification of serum PON $1^{(29)}$. PON 1 is not present in low density lipoprotein (LDL) or very low density lipoprotein (VLDL), indicating a specific interaction with HDL, may be due to apo A1. The protection offered by PON 1 against atherosclerosis is proved as atherosclerotic lesions reduced in human PON1transgenic mice. ${ }^{(30)}$

Association of PON 1 with HDL through apo a1. There are two major and common polymorphisms found in the PON 1coding region. First glutamine $\rightarrow$ arginine substitution at position 192 i.e.(Q192R), and second leucine $\rightarrow$ methionine substitution at position 55 leadingto (L55M). ${ }^{(31)}$ The L55M polymorphism affects the PON 1 protein concentration and Q192R greatly affects the PON 1 activity.PON 1 plays a crucial role in metabolizing certain xenobiotics. But as some of these compounds do not occur in nature, the question which is still unanswered is regarding the natural substrate for Paraoxonase 1 and is the possible physiological role attributed to PON $1 .^{(32)}$ The all three PONs playsan important role in maintaining low oxidative stress and therefore in the prevention of atherosclerosis. ${ }^{(33)}$

The menopausal stage is associated with hormonal and metabolic changes. The menopause associated dyslipidemia and increased oxidative stress are thought to be the frontrunners. The small dense LDL, the most atherogenic subclass was revealed to be increased in postmenopausal women. The antioxidant HDL associated enzyme paraoxonase 1 proved to be decreased after menopause. Hence we are examining, the relation between sdLDL-C concentration and PON1 activity with menopause and, whether these two factors are the most important determinants for the increased CHD risk in postmenopausal women.

\section{Aims and Objectives}

The aims and objectives of present study are,

1) To estimate the small dense LDL (sdLDL) cholesterol levels in postmenopausal women

2) To estimate the Paraoxonase 1 activity in postmenopausal women.

3) To correlate the sdLDL -C levels, PON1 activity with menopause.

The parameters analyzed in the study are,

1) Serum small dense LDL cholesterol

2) Serum Paraoxonase 1 activity

3) Serum total cholesterol

4) Serum triglyceride

5) Serum high density lipoprotein cholesterol

6) Serum very low density lipoprotein cholesterol

7) Serum low density lipoprotein cholesterol

\section{Material and Methods}

The present study was carried out at Medical College hospital. Patients attending OPD of this hospital were selected. A total 80 female subjects were included in this study. These 80 subjects were divided into cases, consists of 40 postmenopausal women and controls consists of 40 healthy premenopausal women from the general population, having same socio-economic conditions as that of the cases. Blood Collection: Fasting blood sample was collected from the subjects. Blood was allowed to clot and then centrifuged for serum separation. The serum was used for estimation of sdLDL-C, PON1 activity and Lipid profile. The serum variables were analyzed using ERBA smart lab Auto analyzer. 


\section{JMSCR Vol||06||Issue||07||Page 831-842||July}

\section{Observation and Result}

The present study was carried out at medical college hospital. Total 80 women were enrolled for the study. These 80 women were then divided into two groups, 40 postmenopausal cases, and 40 healthy premenopausal controls. The parameters studied were Small dense LDL- cholesterol (sdLDL-C)
Paraoxonase 1activity

Total cholesterol,

Triglyceride,

HDL-Cholesterol,

VLDL-Cholesterol,

LDL-Cholesterol

The results of cases were compared with controls.

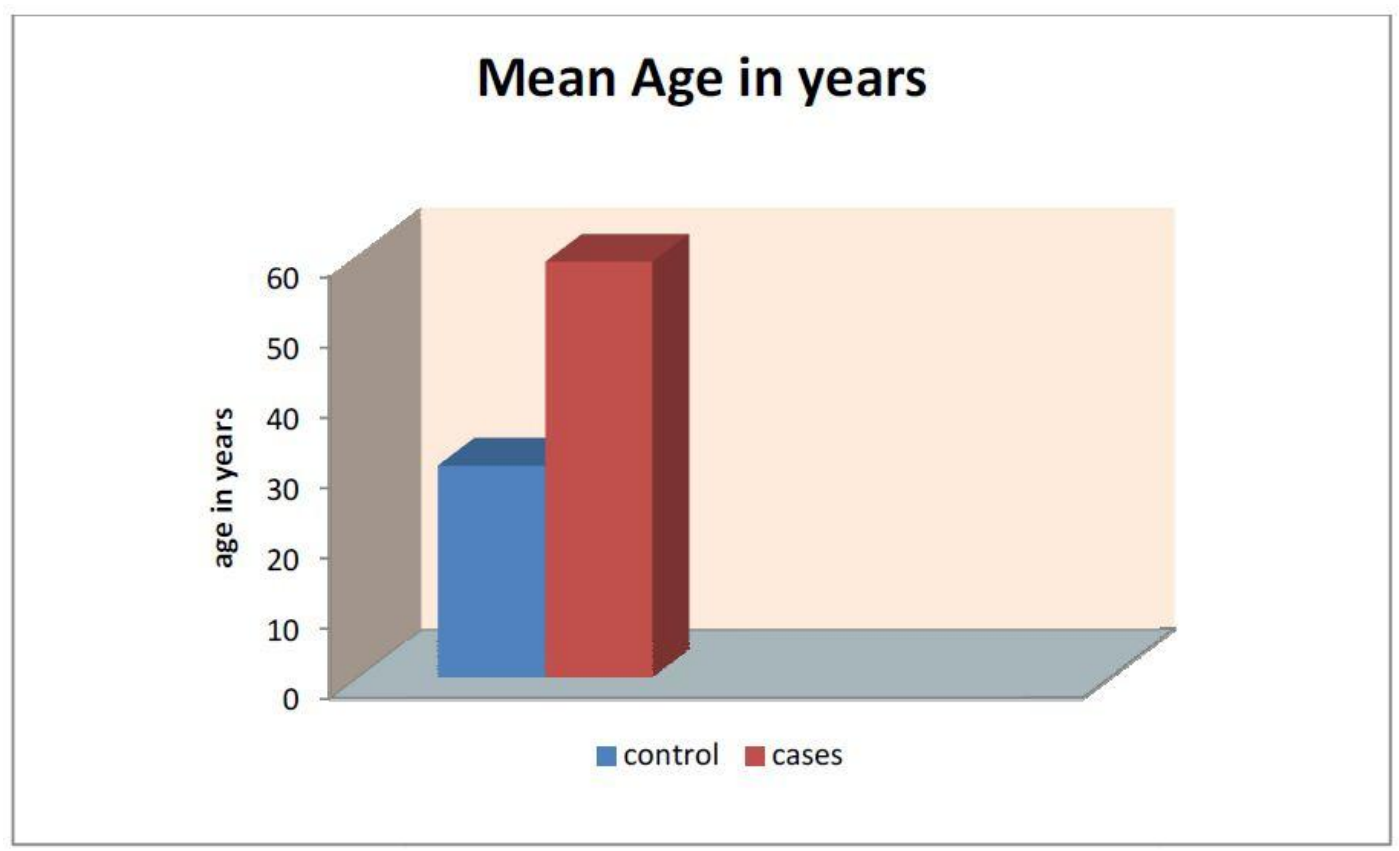

Table 1: Mean age of controls and cases

\begin{tabular}{|l|c|c|}
\hline & Meanage (years) & Standard deviation \\
\hline Controls(no.40) & 30.12 & 7.20 \\
\hline Cases(no.40) & 59.1 & 10.04 \\
\hline
\end{tabular}

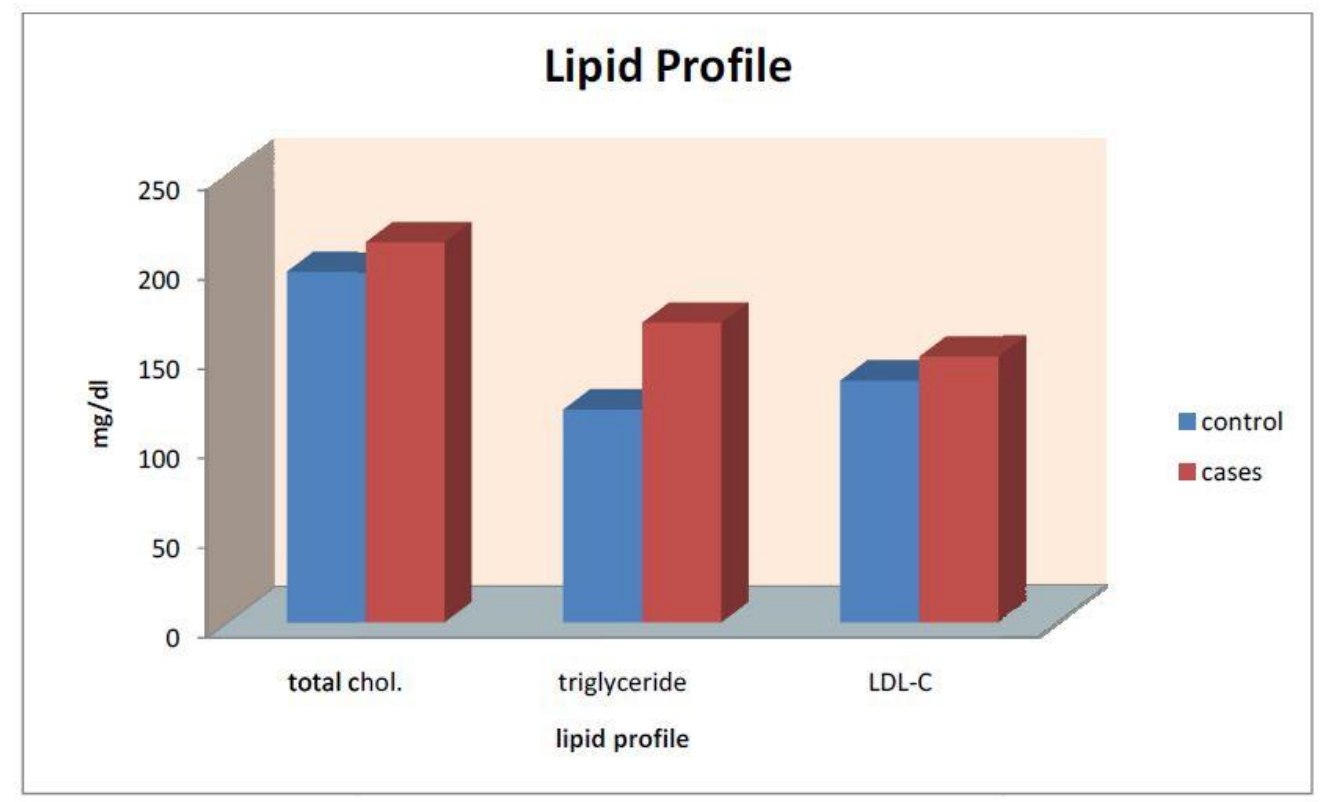


Table 2 Serum Lipid profile in controls and cases

\begin{tabular}{|l|c|c|}
\hline & $\begin{array}{c}\text { Controls } \\
\mathrm{mg} / \mathrm{dl}(\text { S.D. })\end{array}$ & $\begin{array}{c}\text { Cases } \\
\mathrm{mg} / \mathrm{dl}(\text { S.D. })\end{array}$ \\
\hline Total Cholesterol & $195.35 \pm 36.61$ & $212.37 \pm 48.43$ \\
\hline Triglyceride & $118.95 \pm 43.41$ & $167.52 \pm 68.79 *$ \\
\hline LDL-C & $135.12 \pm 34.52$ & $148.55 \pm 40.54$ \\
$* P<0.05$ &
\end{tabular}

The Triglyceride levels in cases $(167.52 \pm 68.79)$ are increased significantly $(p<0.05)$ when compared with controls (118.95 \pm 43.41$)$. The serum levels of total cholesterol (212.37 \pm 48.43 vs.195.35 \pm 36.61$)$ and LDL-C $(148.55 \pm 40.54$

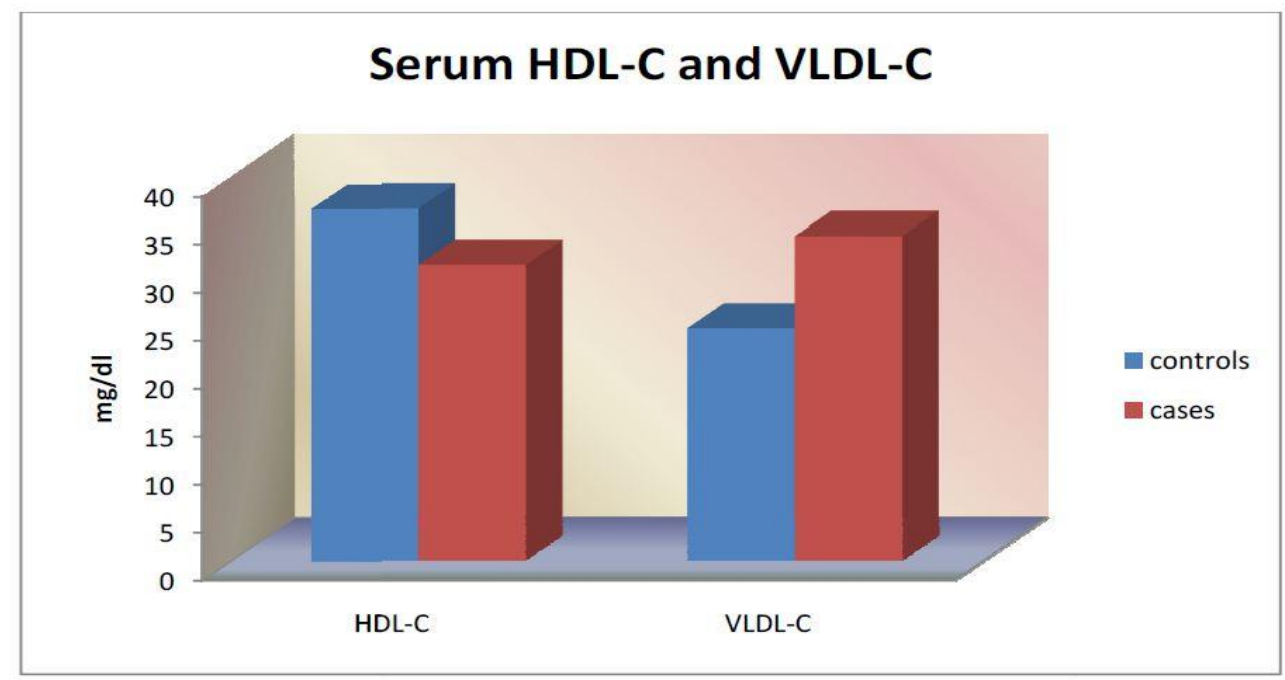

Table3: Serum HDL-C and VLDL-C in controls and cases.

\begin{tabular}{|c|c|c|c|}
\hline & Controls & Cases & Pvalue \\
\hline HDL-C & $36.67 \pm 7.94$ & $30.92 \pm 7.47$ & $<0.05$ \\
\hline VLDL-C & $24.3 \pm 7.94$ & $33.85 \pm 14.89$ & $<0.05$ \\
\hline
\end{tabular}

The serum HDL-C levels are decreased significantly $(\mathrm{P}<0.05)$ in cases $(30.92 \pm 7.47)$ when compared with controls $(36.67 \pm 7.94)$. The VLDL-C levels are increased significantly $(\mathrm{P}<$ $0.05)$ in cases $(33.85 \pm 14.89)$ when compared with controls $(24.3 \pm 7.94)$

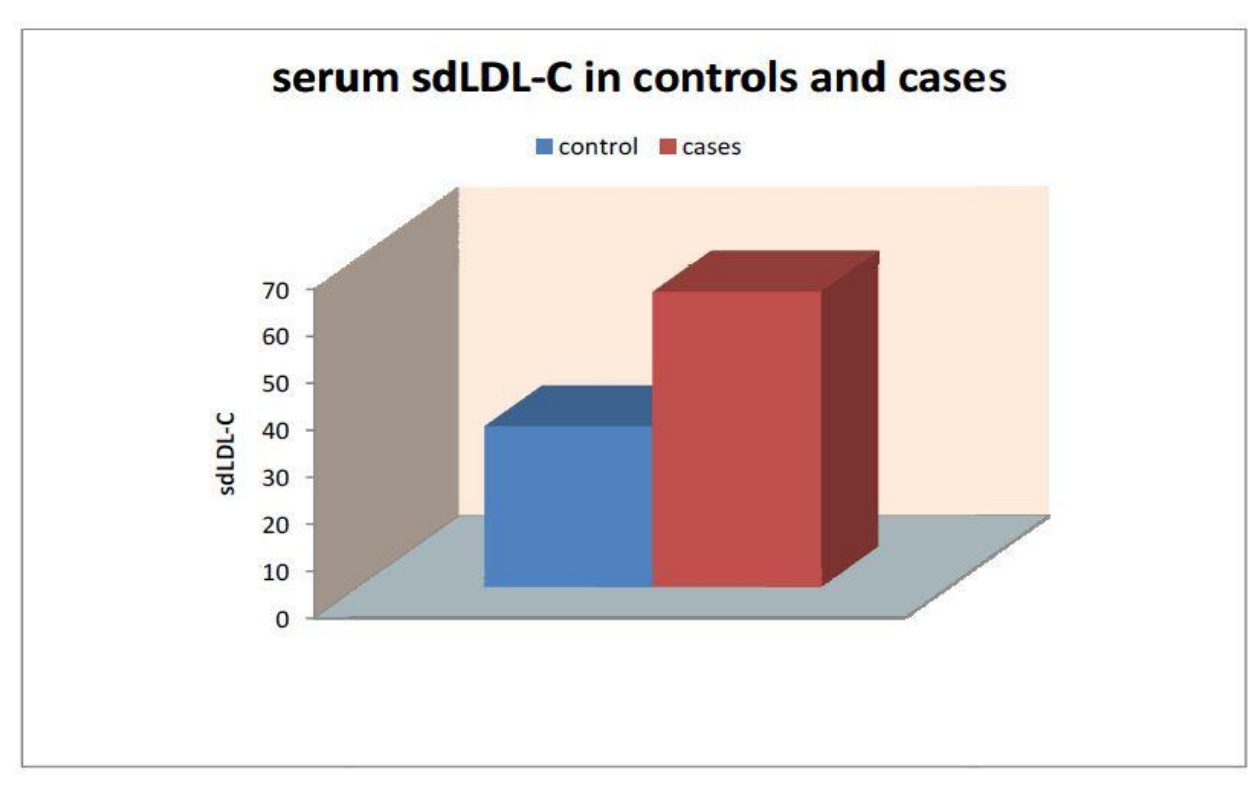




\section{JMSCR Vol||06||Issue||07||Page 831-842||July}

Table 4: Serum sdLDL-C levels in controls and cases.

\begin{tabular}{|c|c|c|c|}
\hline & Controls & Cases & $P$ value \\
\hline sdLDL-C & $34.25 \pm 14.39$ & $62.87 \pm 17.90$ & $<0.05$ \\
\hline
\end{tabular}

The sdLDL cholesterol levels in cases $(62.87 \pm 17.90)$ are significantly increased as compared to controls (34.25 \pm 14.39$)$.

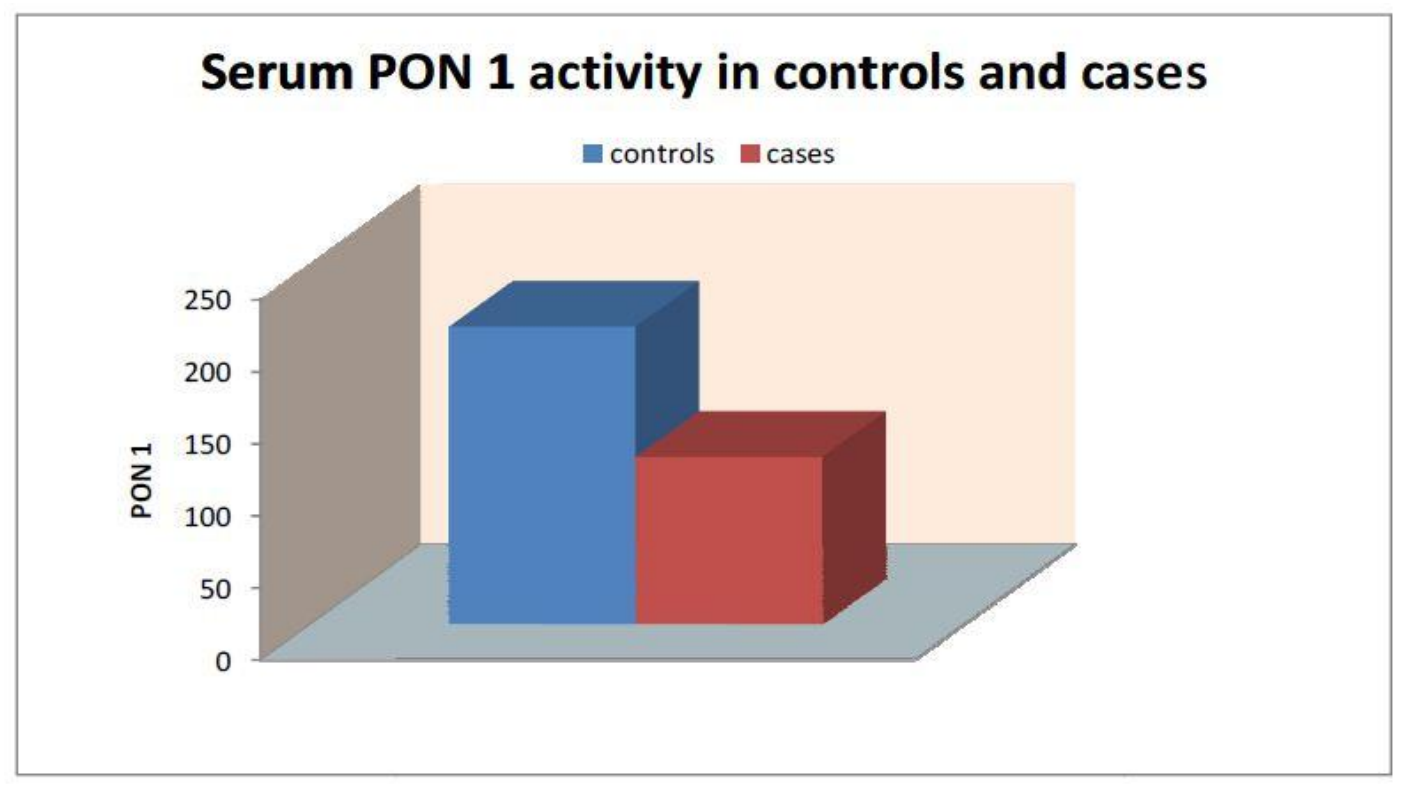

Table5: Serum Paraoxonase1 activity in controls and cases.

\begin{tabular}{|c|c|c|c|}
\hline & Controls & Controls & Pvalue \\
\hline Paaoxonase1(U/L) & $206.55 \pm 69.05$ & $116.16 \pm 76.59$ & $<0.05$ \\
\hline
\end{tabular}

Paraoxonase 1 activity in cases $(116.16 \pm 76.59)$ is significantly decreased as compared to controls $(206.55$ $\pm 69.05)$.

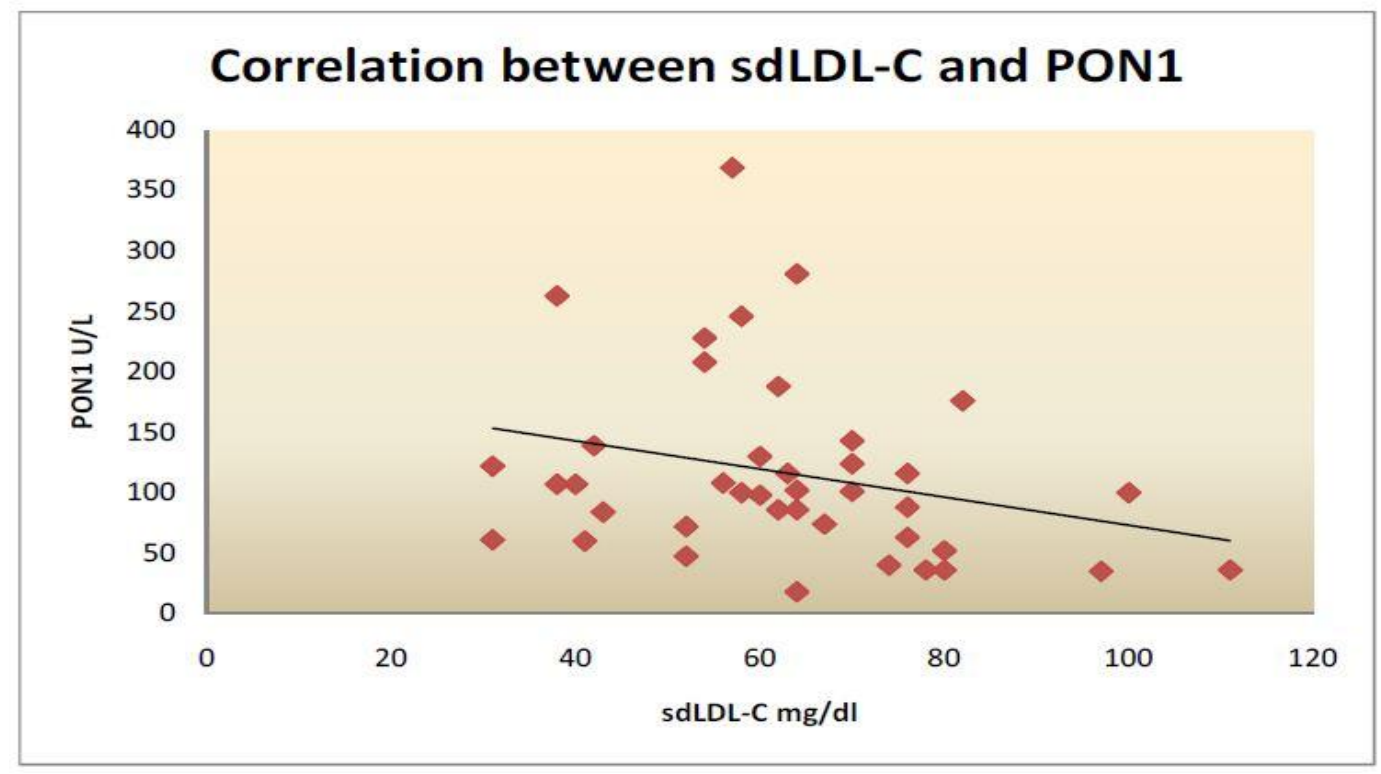

Correlation coefficient: $\mathrm{r}=-0.271 ; \mathrm{P}<0.01$

There is significant negative correlation between small dense LDL cholesterol and paraoxonase1. 


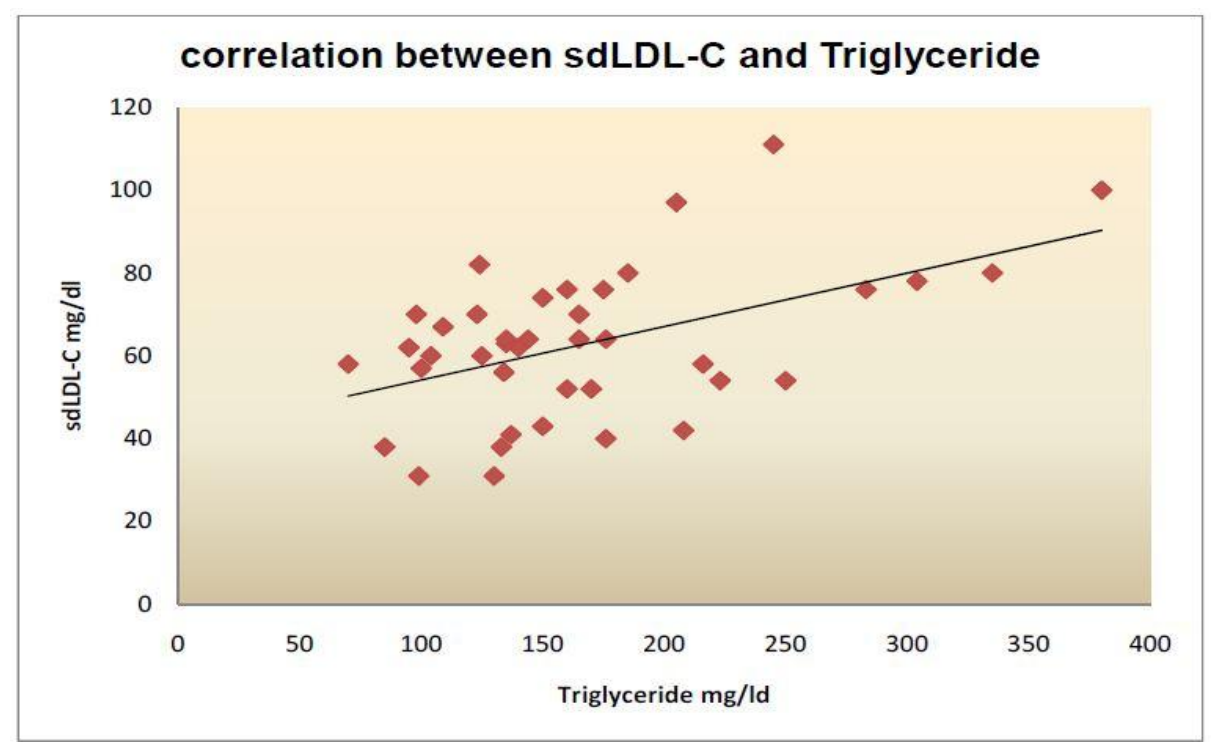

Correlation coefficient, $r=0.496 \mathrm{P}<0.001$

There is significant positive correlation between triglyceride and sdLDLC levels in cases.

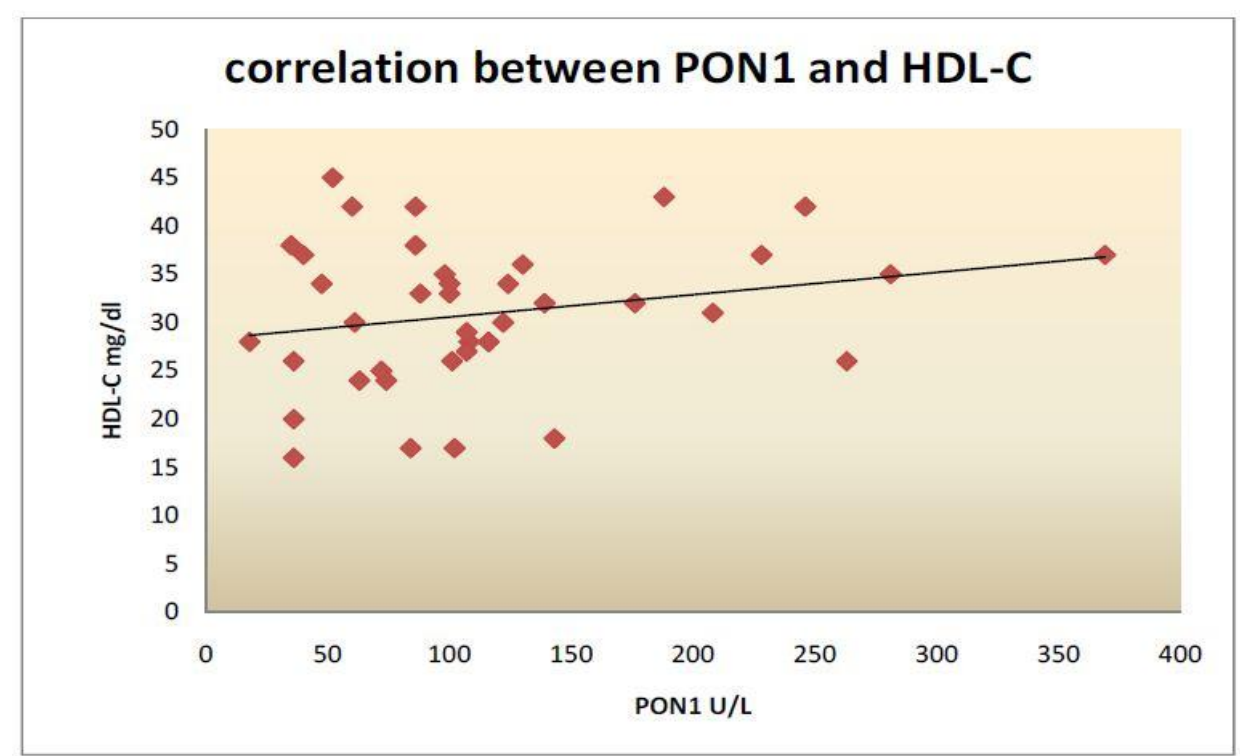

Correlation coefficient, $\mathrm{r}=0.237 \mathrm{P}<0.05$

There is significant but weak positive correlation between paraoxonase 1 activity and HDL-C levels in case

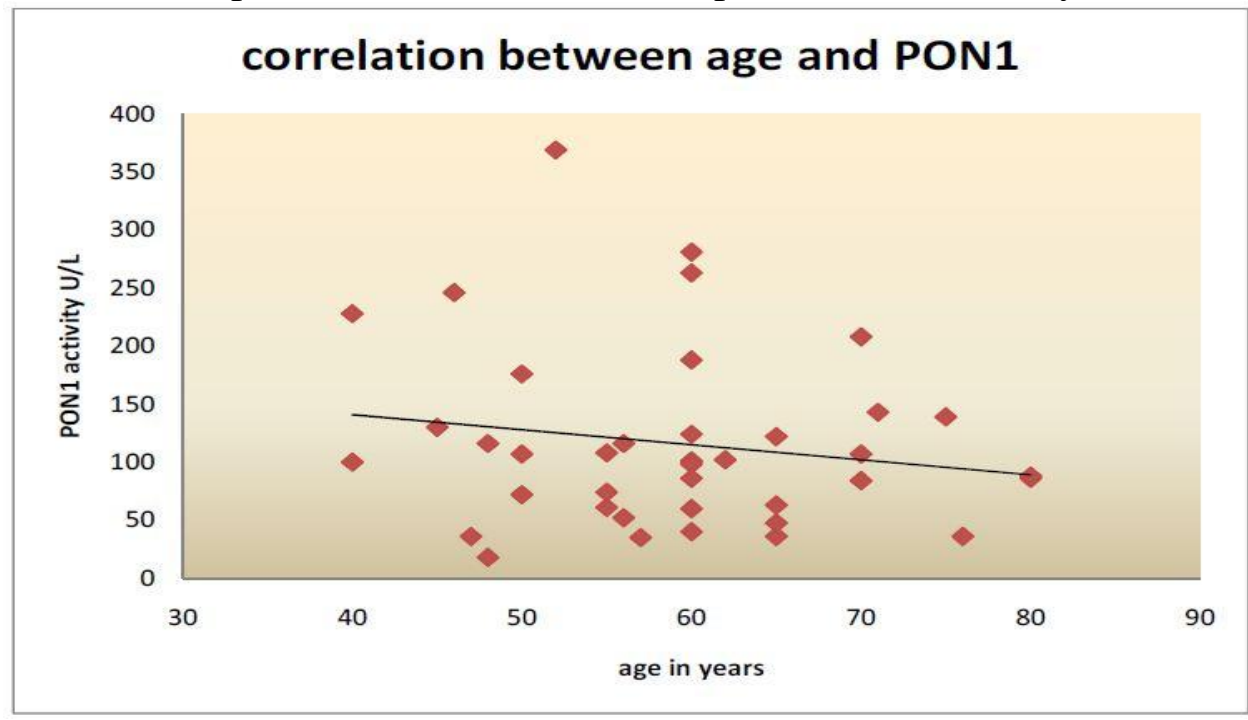

The serum paraoxonase 1 activity is significantly negatively correlated with the age in cases 


\section{Discussion}

Menopause is associated with many hormonal and metabolic changes ${ }^{(23)}$. alteration in lipoprotein profile is one of them. dyslipidemia in postmenopausal women is characterized by increased levels of circulating total cholesterol, triglyceride, VLDL-C, LDL-C mainlysd LDL -C, subsclass of LDL and decreased HDL-C levels. (Present study clearly shows the menopause related changes in the lipoproteins with increase in triglyceride, mainly sdLDL-C, and decrease in HDL sdLDL-C levels in postmenopausal women. The decreased antioxidant defense due to decreased activity of PON1 further deteriorates the condition leading to increased risk for CHD in postmenopausal women. Present study clearly shows the menopause related changes in the lipoproteins with increase in triglyceride, sdLDL$\mathrm{C}$ and decrease in HDL-Cleves in postmenopausal women. The decreased antioxidant defense due to decreased activity of PON1 further deteriorates the condition leading to increased risk for CHD in postmenopausal women. Circulating total cholesterol, triglyceride, VLDL-C, LDL-C mainly sdLDL-C, subclass of LDL and decreased HDL-C levels. ${ }^{(22)}$ Due to these changes, postmenopausal women are more prone to develop CHD than the premenopausal. The increased levels sdLDL-C, of are characteristic of menopause. ${ }^{(27)}$ The sdLDL is a subtraction of LDL with smallest size and largest density. Duet the small size, these particles retained for longer time in circulation and can easily penetrate the vascular endothelium. These features make sdLDL the most atherogenic lipoprotein subclass and its increased levels can signify the increased risk of atherosclerosis. ${ }^{(26)}$ Paraoxonase 1 is a HDL associated enzyme. The antioxidant property of HDL is mainly due to PON1. The PON1 inhibits the LDL and HDL oxidation and therefore is a strong protective and anti atherosclerotic enzyme. ${ }^{(20)}$ The present study was carried out in a medical college hospital. Total40 postmenopausal women were enrolled as cases. These cases were compared with 40 healthy premenopausal women. The parameters studied were small dense LDL-C, paraoxonse1 activity, and lipid parameters (Total cholesterol, triglyceride, HDL-C, LDL-C, and VLDL-C). The present study, demonstrate significant changes with lipoproteins unhealthy postmenopausal women. There is significant increase in triglyceride levels with decreased HDL-C levels in postmenopausal women. These changes in postmenopausal women are accompanied with the significant increase in sdLDL-C, which is the most atherogenic. Smaller and denser subclass of LDL and decrease in serum activity of PON1 can protect both LDL and HDL from oxidation. in present study serum concentration of total cholesterol and LDL cholesterol does not differ between pre and post menopausal women. This suggests, when only quantitative aspect of cholesterol metabolism are considered, it does not show any disturbances due to menopause. Thus, though LDL-C is the primary risk factor for CHD, the postmenopausal women not appear to have increased risk. However, when qualitative modifications fold sdLDL-C are taken into account, it shows a completely different picture. The postmenopausal status appears to be associated with presence of increased sdLDL-C, This sdLDL-C, is a proven risk factor for the coronary disease. The increased level of triglyceride $(167.52 \pm 68.79$ vs. $118.95 \pm$ $43.41 \mathrm{mg} / \mathrm{dl})$ and decreased HDL-C (30.92 \pm 7.47 vs. $36.67 \pm 7.94 \mathrm{mg} / \mathrm{dl}$ ) strongly reflect the estrogen deficiency state. The effect of menopausal transition 46 state over lipoprotein metabolism was shown by Matthews K.A. ${ }^{(30)}$ in 541women who followed over 2.5 years. Similarly, Jensen $J,{ }^{(31)}$ in a 2-3 year longitudinal study of initially premenopausal women, found that the serum levels of total cholesterol, triglyceride and LDL-C were significantly increased while HDL-C levels were decreased in postmenopausal women. Findings of these studies show the acute effects of estrogen deficiency at premenopausal period during transition from pre to post menopause. Age is an important and independent irreversible risk factor for 
atherosclerosis and CHD. But Stevenson $\mathrm{J} \mathrm{C}^{(32)}$ in the study of 542 healthy postmenopausal women showed age, BMI and other potential confounding variable independent changes in the midst of lipoproteins with higher concentration of total cholesterol (increase of 14\%) serum triglyceride (12\% increase), LDL-cholesterol (27\% increase), and lower concentration of HDL2-cholesterol (27\% decrease) as compared to premenopausal women, underlining the important role played by menopausal transition in determining the CHD risk in postmenopausal women, Age is an important and independent irreversible risk factor for atherosclerosis and CHD as per our study. These findings were further strengthened by the observations of Poehlman E $\mathrm{T}^{(33)}$ showing a significant decrease in HDL-C levels and47 increase in serum levels of LDL-C and fasting triglyceride level in 38 healthy middle aged women during their transition from pre to post menopause over 6year period. These lipoprotein changes are associated with decreased concentration of estrogen after menopause ${ }^{(21)}$. In present study the sdLDL Cholesterol levels in cases $(62.87 \pm 17.90)$ were increased significantly as compared with controls $(34.25 \pm 14.39)$. The finding is similar to the study of Elena vatic et $a l^{(27)}$ which is the most closely matched study to present study. Ernst J Schaefer ${ }^{(22)}$ like us found that the peak particle size of LDL was reduced significantly in postmenopausal women48than the premenopausal women. The increased concentration of sdLDL-C in present study which is associated with no change in total cholesterol and LDL-C levels suggest the qualitative change in lipoproteins after the menopause. The higher levels of sdLDL which has proven atherogenic potential, ${ }^{(34,35)}$ with increased triglyceride and decreased protective HDL-C contribute to increased risk orchid after the menopause. The positive correlation $(\mathrm{r}=0.496, P<0.001)$ between sdLDL -C levels with serum triglyceride concentration is very much analogous with the study of McNamara et al $^{(36)}$ Akihiko Takatsuki ${ }^{(25)}$ also found the similar results with increased
sdLDL particles with hypertriglyceridemia in postmenopausal women. The increased concentration of sdLDL in postmenopausal women also points towards the role of estrogen in the synthesis and metabolism of these particles. The Estrogen therapy after menopause shown to reduce the concentration of $\operatorname{sdLDL}^{(24)}$ in the circulation but the precise mechanism of this reduction has not been evaluated The genetic inheritance theory put forwarded by Austin M $\mathrm{A}^{(37)}$ also support our finding of increased concentration of sdLDL-C in postmenopausal women. Study shows among postmenopausal women and among men over 20 years of age, $44 \%$ of population expected to express the predominance of sdLDL. The phenotypic expression of sdLDL can be affected by age. The significantly decreased paraoxonase 1 activity in postmenopausal women (116.16 \pm 76.59) compared to premenopausal (206.55 \pm 69.05) suggest decreased antioxidant defense after menopause. The decreased levels of HDL-C in our study are significantly related to decreased activity of PON1. Our results are very much similar to the findings by Selahattin Kumru et al (29), with decreased activity of PON1 in elderly women compared to younger. The postmenopausal decrease of estrogen may indirectly affect the PON1 concentration and activity. The postmenopausal decrease inPON1 activity was also seen in the study of Ata Topcuoglu. An age dependency of PON1 is clearly seen in present study, showing strong negative correlation with age $(r=-0.348)$. Lidice Seers et $\mathrm{al}^{(28)}$ also found the similar correlation in the elderly women. Present study clearly shows the menopause related changes in the lipoproteins with increase in triglyceride, sdLDL-C and decrease in HDL-Cleves in postmenopausal women. The decreased antioxidant defense due to decreased activity of PON1 further deteriorates the condition leading to increased risk for CHD in postmenopausal women. Study the strong negative correlation $(P<0.01)$ found between the serum levels of sdLDL-C and PON1 activity. 
The present study is an observational study, it has major and distinctive strengths including contemplated to be first the study in postmenopausal women determining the effect of menopause sdLDL-C and PON1, the atherogenic tendency is judged by more specific LDL subclass sdLDL. We have not done the serum estrogen levels, which will restrict our conclusion. Further larger studies are required to confirm the results.

\section{Conclusion}

The present study demonstrates that the postmenopausal period is associated with significant changes in lipoprotein like increased triglyceride and decreased HDL-C concentration. Furthermore these changes are accompanied by increased sdLDL-C and decreased protective PON1. The decreased activity of PON1 could be due to lower levels of HDL-C. The decreasing PON1 activity was also found to relate with increased sdLDL these alterations in postmenopausal women suggest increased risk for CVD.

\section{Bibliography}

1. Harrison's principles of medicine, 17 Edition Vol II, Ed. Anthony S Fauci..Pathogenesis, prevention and treatment of atherosclerosis. Page 1501 Mc - Graw Hill. 2008.

2. Park's Textbook of PSM, 20th edition, K Park, Atherosclerosis, non communicable diseases, page 316-317. Bhanot publication.

3. Sybil L Crawford, C B Johannes. The epidemiology of cardiovascular diseases in postmenopausal women. J. Clin. Endocrinol. Metab.1999;84: 1803-1806

4. Third Report of the National Cholesterol Education Program (NCEP) Expert Panel on Detection, Evaluation, and Treatment of High Blood Cholesterol in Adults (Adult Treatment Panel III) Final Report. Circulation 2002;106;3143
5. Yan Feng, Xiumei Hong, ElissaWilker et al, Effect of age of menarche, reproductive years, and menopause on metabolic risk factors for cardiovascular diseases. Atherosclerosis 2008;196; 2: 590-597

6. Tremollieres F A, Pouilles J M, cauneille C, Ribot C. Coronary heart disease risk factors and menopause: a study in 1684 French women. Atherosclerosis, 1999; 142: 415- 423.

7. Selby J V, Austin M A,Newman B, Zhang $\mathrm{D}$ et al. LDL subclass phenotype and the insulin resistance syndrome in women. Circulation 1993; 88: 381-387.

8. J George fodor, rayka Tzerovska. Coronary heart disease: is gender important? The $J$ of men health and gender; 2004;1(1):32-37 57

9. Yen Y Tan, et al, gender differences in risk factors for coronary heart disease. Maturitas2010;65:149-160

10. Yichuan Wen et al, Effect of menopause on low density lipoprotein oxidation:is estrogen an important determinant? Maturitas 2000;34:233-8

11. Valeria zago, Silvia S. Brites F. Berg G et al. Impaired HDL antioxidant activity in healthy postmenopausal women. Atherosclerosis, 2004; 177: 203-210.

12. Matthias Barton, Mark M Kockx. Estrogen and apoptosis in atherosclerosis. International Congress series, 2002; 1229: 81-93

13. Karen Heun, kim Harley, Jordan Brooks, Alan Hubbard, Asa Bradman Brenda Eskenazi and Nina Holland. Developmental changes in PON1 enzyme activity in young children and effects of PON1 polymorphism. Environ. Health Perspect 2009; 117:1632-1638.

14. Michael I Mackness and Colin H Walker. Multiple forms of sheep serum A- esterase activity associated with the high density lipoprotein. Biochem J. 1988; 250: 539545. 
15. Bert N La Du, Michael Aviram, Scott Billecke, Mohamad Navab and et al. On physiological role(s) of the paraoxonases. Chemico- biological Interactions 1999; 119-120: 379-388.

16. Aaron Tward, Yu Rong Xia, Xu Ping Wang, Yi Shou Shi and et al. Decreased Atherosclerosis lesion formation in human serum Paraoxonase transgenic mice. Circulation.2002; 106:484-490.

17. Adkins S, Gan KN, Mody M. Molecular Basis for the Polymorphic Forms of Human Serum Paraoxonase/Arylesterase: Glutamine or Arginine at Position 19 1, for the Respective A or B Allozymes. Am. $J$ Hum Genet 1993;52:598-608

18. Rainwater D L, Sue Rutherford, Dyer T D, Rainwater E $\mathrm{D}$ et al.determinants of variation in human serum Paraoxonase activity. Heredity 2009;102 (2): 147- 154

19. Mackness B, mackness M I, Arrol S Turkie W et al. Effect of thehuman serum Paraoxonase 55 and 192 genetic polymorphisms on theprotection by high density lipoprotein against low density lipoprotein oxidative modification. FEBS Letters 1998;423:57-60

20. Kasper K Berneis, krauss R M. Metabolic origin and clinical significance35. J Lipid Res 2002;43: 1363-1379

21. Jane A. Cauley, James P. Gutai, Nancy W Glynn, Mandeline P, Cottington E, Kuller L.H. Serum estrone concentration and coronary artery disease in postmenopausal women. Arterioscler.Thromb.1994;14: 1428

22. Ernst J. Schaefer, Stefania L. Cohn S, Schafer M, Ordovas J, castelli W. et al Effects of age, gender, and menopausal status on plasma low density lipoprotein cholesterol and apolipoprotein B levels in the Framingham offspring study. J. lipid Res. 1994;35: 779-79223) Domenico de Aloysio, Gambacciani M. Meschia M, Pansini F, Modana A.B, Bolis P. F, et al
The effect of menopause on blood lipid and lipoprotein levels. Atherosclerosis, 1999; 147: 147- 153.

23. Mavis Abbey, Alice Owen, Michio Suzakawa, Paul Roach, Paul Nestel. Effects of menopause and hormone replacement therapy onplasma lipids, lipoproteins and LDL receptor activity Maturitas 1999;33: 259-269.

24. Akihiko Wakatsuki, Nobuo Ikenoue, Yuji Okatani, Takao Fukaya. Oestrogen induced small low density lipoprotein particles may beatherogenic in postmenopausal women. J. Am. Coll. Cardiol.2001;37:425-430

25. Sartipy P. Camejo G, Stevensson L, Camejo E. Phospholipase A2modification of LDL forms small high density particles with increased affinity for proteoglycans and glycosaminoglycans. J. Bio. Chem.1999;274 (36):25913-25920..

26. Vekic J, Zeljkovic A, Ivanovic Z, Kalimanovska V, Stanojevic N,Memon L et al. Small dense LDL cholesterol and apolipoprotein B:relationship with serum lipids and LDL size.Atherosclerosis2009; 207: 496-501.

27. Seres I, Paragh G, Deschene E, Fulop T, Khalil A. Study of factors influencing the decreased HDL associated PON1 activity with aging. Experimental Gerontology 2004; 39: 59-66.

28. Kumru S, Aydin S, Aras A, Gursu M, Gulcu F. Effects of surgical menopause and oestrogen replacement therapy on serumparaoxonase activity and plasma malondialdehyde concentration. Gynec. Obste. Invest. 2005; 59:108-112.

29. Matthews K A, Meilahn E, Kuller L H, Kelsey S F, Caggiula A W, WingR R. Menopause and risk factors for coronary heart disease. N Eng JMed 1989; 321: 641646.

30. Jensen J, Nilas L, Christiansen C. Influence of menopause on serumlipids 
and lipoproteins. Maturitas 1990; 12 (4): 321-331.

31. Stevenson J C, Crook D, Godsland I F. Influence of age and menopause on serum lipids and lipoproteins in healthy women.Atherosclerosis.1993; 98: 83-90.

32. Poehlman E T, Toth M J, Ades PA, Rosen C J. Menopause associated changes in plasma lipids, insulin like growth factor I and blood pressure: a longitudinal study. Eur J Clin Invest 1997; 27 (4):322-326. .

33. Hannia Campos, Judith R. Mcnamara, Peter W. F. Wilson, Jose M.Ordovas Ernst J. Schaefer. Differences in low density lipoprotein subfractions and apolipoproteins in premenopausal and postmenopausal women. J. Clin. Endocrinol.Metab.1988; 67: 30-35.

34. Krauss R M. Dense low density lipoproteins and coronary arterydisease. Am J Cardiol. 1995; 75: 53B - 57B.

35. McNamara J R, Jenner J L, Li Z, Wilson P W, Schaefer E J. Change inLDL particle size is associated with change in plasma triglyceride concentration. Arteriscler ThrombVascBiol1992; 12: 1284 - 1290.

36. Austin M A, King M C, vranizan K M, Newman B, Krauss R M .Inheritance of low density lipoprotein subclass patterns: results of complex segregation analysis. Am J Hum Genet 1988; 43: 838 - 846. 